\title{
Conditions d'utilisation des méthodes automatiques de dénombrement des cellules du lait : étalonnage et conservation des échantillons de lait
}

\author{
par \\ R. GRAPPIN et R. JEUNET \\ I.N.R.A., Station Expérimentale Laitière de Poligny (Jura)
}

Tous les travaux portant sur les méthodes automatiques ou semi-automatiques de comptage des cellules du lait (Compteur-Coulter, Auto-Analyser Fossomatic) montrent que ces méthodes sont suffisamment précises pour être utilisées aussi bien dans le cadre d'une prophylaxie des mammites que pour définir la qualité hygiénique ou payer le lait en fonction de sa teneur en cellules.

Les normes d'utilisation existantes, établies notamment pour le Compteur-Coulter, doivent permettre aux laboratoires de donner des résultats suffisamment répétables. Toutefois, pour que ces résultats soient comparables entre laboratoires, il faut non seulement que les modes opératoires soient normalisés mais aussi et surtout que les techniques d'étalonnage soient bien définies. Ceci est particulièrement vrai dans le cas des méthodes entièrement automatisées pour lesquelles l'étalonnage est certainement le facteur le plus important influençant la précision des résultats.

Les appareils représentant souvent des inv stissements importants, les laboratoires doivent les utiliser au maximum de leur capacité. Pour cela, les analyses sont faites dans des laboratoires centraux regroupant un grand nombre d'échantillons de toute nature (laits individuels ou laits de troupeaux) et de provenances géographiques parfois éloignées du lieu d'analyse. Dans ces conditions, il est souvent difficile sinon impossible d'effectuer des analyses sur des échantillons de lait frais âgés de moins de $24 \mathrm{~h}$. En fait, pour limiter les frais de prélèvement et de collecte des échantillons, les numérations cellulaires se font souvent sur des échantillons destinés aux dosages de matière grasse et de matière azotée ; ces échantillons sont le plus souvent additionnés d'un produit de conservation afin d'être analysés au laboratoire plusieurs jours après le prélèvement. 
Dans ce travail, nous nous proposons d'étudier l'étalonnage et les conditions de conservation des échantillons de lait, en nous limitant aux seuls méthodes et appareils actuellement utilisés en France : le Compteur Coulter et le Fossomatic.

\section{MATERIELS ET METHODES}

\section{1) Echantillons de laits examinés}

Les essais sont tous faits à partir de laits de troupeaux prélevés au plus tard $12 \mathrm{~h}$ après la traite, le lait étant, entre temps, conservé à $4^{\circ} \mathrm{C}$. Tous les échantillons proviennent de laits de bonne qualité bactériologique (teneur en germes microbiens inférieure à $300000 / \mathrm{ml}$ de lait). Lorsque les échantillons sont additionnés d'un produit de conservation, les doses employées sont les suivantes : $\mathrm{K}_{2} \mathrm{Cr}_{2} \mathrm{O}_{7}$ : 1 p. $1000, \mathrm{HgCl}_{2}$ : 0,7 p. $1000, \mathrm{NaN}_{3}$ : 0,3 p. 1000 .

\section{2) Numérations cellulaires}

Les numérations sont faites :

a) suivant la méthode microscopique de Prescott et Breed déjà décrite (Grappin et Jeunet, 1974),

b) avec l'appareil Fossomatic (Grappin et Jeunet, 1974 ; SchmidtMadsen, 1975),

c) avec le Compteur Coulter en employant soit la version automatique (Milk-Cell Counter, en abrégé : MCC) avec le réactif somasol, soit la méthode classique avec le réactif de Tolle.

Les caractéristiques de ces deux dernières techniques sont les suivantes :

1) Le Milk Cell Counter est un appareil automatique mis au point par la Sté Coultronics France (Grappin et Jeunet, 1973). Il fonctionne dans les conditions suivantes :

- Fixation : addition de $0,3 \mathrm{ml}$, d'une solution de formol à 3,5 p. 100 , à $4,7 \mathrm{ml}$ de lait (soit une concentration finale en formol de 0,21 p. 100) et chauffage au bain-marie exactement $3 \mathrm{mn}$ à $60^{\circ} \mathrm{C}$.

- Réactif de clarification : solution commerciale (Coultronics) de Somasol (appelé précédemment réactif $\mathrm{H}^{+}$). d'Isoton.

- Réactif de dilution : solution commerciale (Coultronics)

La clarification du milieu se fait instantanément à froid en ajoutant 2,08 $\mathrm{ml}$ de Somasol à $44,7 \mu \mathrm{l}$ de lait. Le comptage est effectué après addition de 2,08 $\mathrm{ml}$ d'Isoton.

- Compteur Coulter : modèle $\mathrm{Z}$, sonde de $140 \mu \mathrm{m}$, comptage sur $0,1 \mathrm{ml}$. Le résultat affiché correspond à la moyenne de trois comptages. 
2) Méthode de Tolle : (Zeidler et al., 1968 ; Grappin et Jeunet, 1971):

- Fixation : addition de $0,2 \mathrm{ml}$ d'une solution de formol à 3,5 p. 100 dans $9,8 \mathrm{ml}$ de lait (soit une concentration finale en formol de 0,07 p. 100); chauffage au bain-marie pendant $30 \mathrm{mn}$ à $60^{\circ} \mathrm{C}$.

- Réactif de clarification : solution comprenant $\mathrm{NaCl}$ (7,6 g) ; Ethanol 96 p. $100(125 \mathrm{ml})$, formol à 35 p. $100(10 \mathrm{ml})$, Triton X 100 $(20 \mathrm{ml})$, tampon TRIS ( $3 \mathrm{~g}$ environ), eau distillée (Q.S.P. $1000 \mathrm{ml}$ ). Le $\mathrm{pH}$ doit être de 7,0 . Ajouter $9,7 \mathrm{ml}$ de réactif de clarification à $0,1 \mathrm{ml}$ de lait, puis chauffage au bain-marie $10 \mathrm{mn}$ à $80 \pm 1^{\circ} \mathrm{C}$.

- Compteur Coulter : modèle F, sonde de $100 \mu \mathrm{m}$, comptage sur $0,1 \mathrm{ml}$ d'électrolyte (le résultat donné correspond à la moyenne de deux comptages).

\section{RESULTATS}

\section{1) Etalonnage des appareils}

L'étalonnage des deux appareils Fossomatic et Coulter consiste à déterminer un seuil appelé " seuil de lecture " correspondant à un signal minimum de comptage. Pour le Fossomatic, l'importance du signal est liée à l'intensité de la lumière fluorescente émise par le complexe ADN-colorant ; pour le Coulter, elle est proportionnelle au volume des particules passant au travers de l'orifice de la sonde. Rappelons qu'il existe deux méthodes pour définir le seuil d'étalonnage :

$1^{\circ}$ Tracer, à partir de l'analyse de plusieurs échantillons de teneurs élevées en cellules, une " courbe de seuil " moyenne, correspondant au diagramme cumulé des comptages effectués par l'appareil en fonction des seuils.

$2^{\circ}$ Comparer sur un nombre suffisamment grand d'échantillons (supérieur à 30 ) les résultats obtenus avec l'appareil et par la méthode microscopique.

Dans le premier cas, le diagramme doit présenter théoriquement un plateau qui se situe entre la zone de bruit et les signaux les plus faibles fournis par les cellules. Le seuil de lecture se situe en principe, au niveau de ce plateau.

En utilisant la méthode de comparaison par rapport au microscope, le seuil de lecture est le seuil pour lequel on a un étalonnage exact, c'est-à-dire une pente de la droite de régression appareil/ microscope égale à 1 et des moyennes égales. Il faut d'autre part, que la précision par rapport à la méthode de référence (caractérisée par l'écart-type résiduel déduit de la régression) soit aussi élevée que possible.

Ces deux méthodes ont été appliquées au Fossomatic et au Compteur Coulter dans le cas d'échantillons de lait, additionnés ou non d'un produit de conservation. 


\subsection{Etalonnage du Fossomatic}

Au cours des essais préliminaires (Grappin et Jeunet, 1974), nous avions d'une part montré la difficulté qu'il y avait à définir le seuil de lecture uniquement d'après la forme de la courbe de seuil et d'autre part constaté que l'addition de produits de conservation modifiait l'allure de cette courbe. Avant de définir le seuil théorique par comparaison avec la méthode microscopique, nous avons entrepris de confirmer ces premiers résultats.

\subsection{Influence de l'addition d'un produit de conservation au} lait sur la courbe de seuil

Trente-trois échantillons de lait ayant entre 50000 et 2,2 millions de cellules $/ \mathrm{ml}$ sont analysés avec le Fossomatic à différents seuils compris entre 0,5 et 2,5. Les analyses sont faites sur des échantillons sans conservateur, et sur les mêmes échantillons immédiatement après addition des différents conservateurs $\left(\mathrm{K}_{2} \mathrm{Cr}_{2} \mathrm{O}_{2}, \mathrm{HgCl}_{2}, \mathrm{NaN}_{3}\right)$.

L'examen des courbes de seuil établies à partir des moyennes géométriques des comptages faits sur les 33 échantillons, confirme d'une part l'absence de plateau bien distinct et d'autre part l'influence de l'addition du produit de conservation sur la forme de la courbe (fig. 1). Les courbes obtenues avec $\mathrm{HgCl}_{2}$ et $\mathrm{Cr}_{2} \mathrm{O}_{7} \mathrm{~K}_{2}$ sont nettement différentes de celles obtenues avec le lait sans conservateur ou avec le lait additionné de NaNs.

Il faut souligner qu'entre les seuils 1,0 et 1,5 les différentes courbes présentent des points d'intersection ou sont très proches les unes des autres, de telle sorte que les moyennes des comptages Fossomatic présentent à chacun des deux seuils des différences non significatives pour les différents conservateurs employés.

\subsection{Comparaison avec les comptages microscopiques}

D'après nos résultats antérieurs (Grappin et Jeunet, 1974) et l'étude des courbes de seuil, il semble que le seuil de lecture doive se situer entre 1,0 et 1,5 .

Sa détermination exacte a été faite à partir de 43 échantillons de lait frais, ayant entre 50000 et 2,0 millions de cellules/ml, examinés au microscope et avec le Fossomatic aux seuils de 1,0 et 1,5. L'examen des résultats montre que la précision des comptages par rapport à la méthode microscopique est identique pour les deux seuils de 1,0 et 1,5. L'écart-type résiduel, exprimé en racine carrée des nombres, est respectivement de 3,00 et 2,76 (différence non significative).

C'est au seuil de 1,5 que la pente de la droite de régression se rapproche le plus de 1 avec des moyennes microscopique et Fossomatic très voisines (fig. 2). 


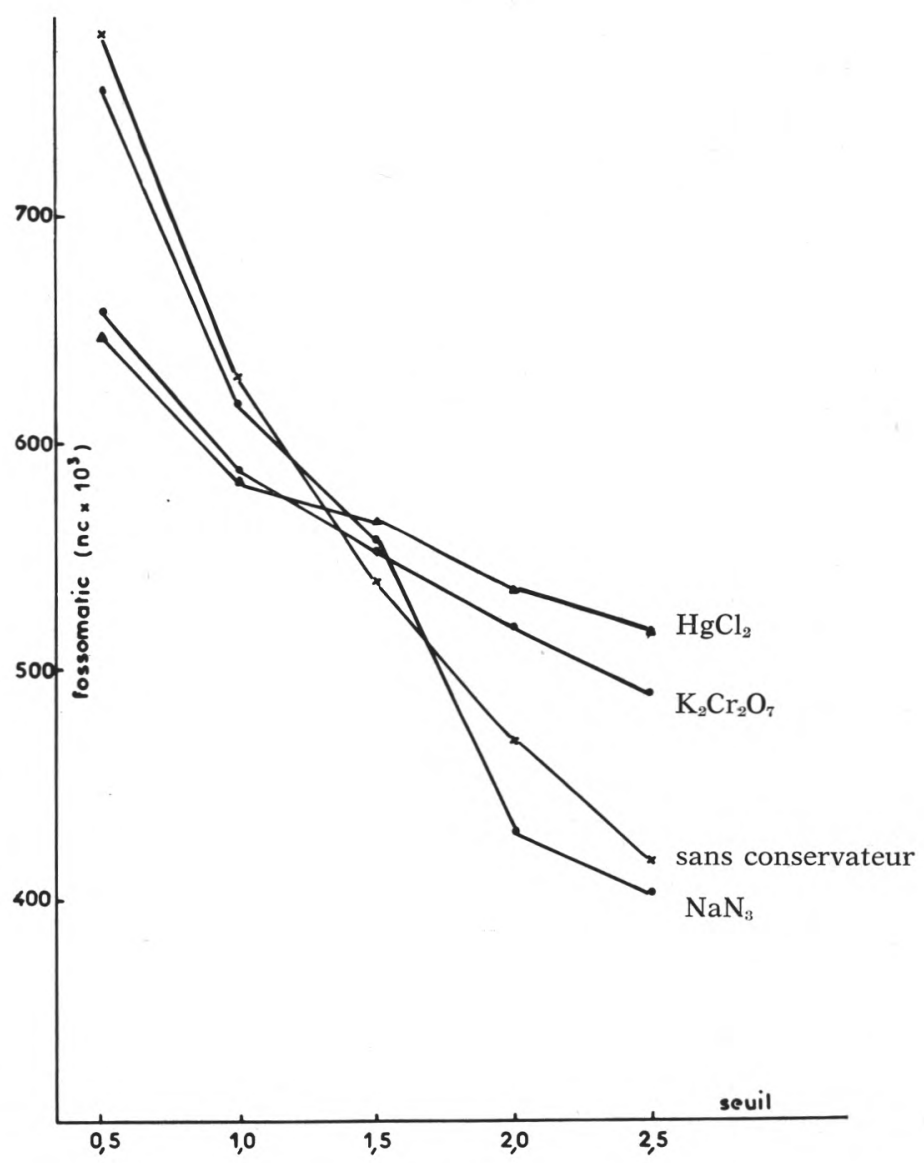

fig. 1

Influence de l'addition d'un produit de conservation au lait sur la courbe de seuil du Fossomatic : diagramme cumulé du nombre de cellules déterminé par l'appareil en fonction du seuil de lecture ( $\mathrm{n}=33$ échantillons).

\subsection{Etalonnage du Compteur Coulter}

L'étude porte plus particulièrement sur le MCC pour lequel le seuil de lecture n'a pas été défini avec précision au cours de notre première étude de l'appareil (Grappin et Jeunet, 1973). 


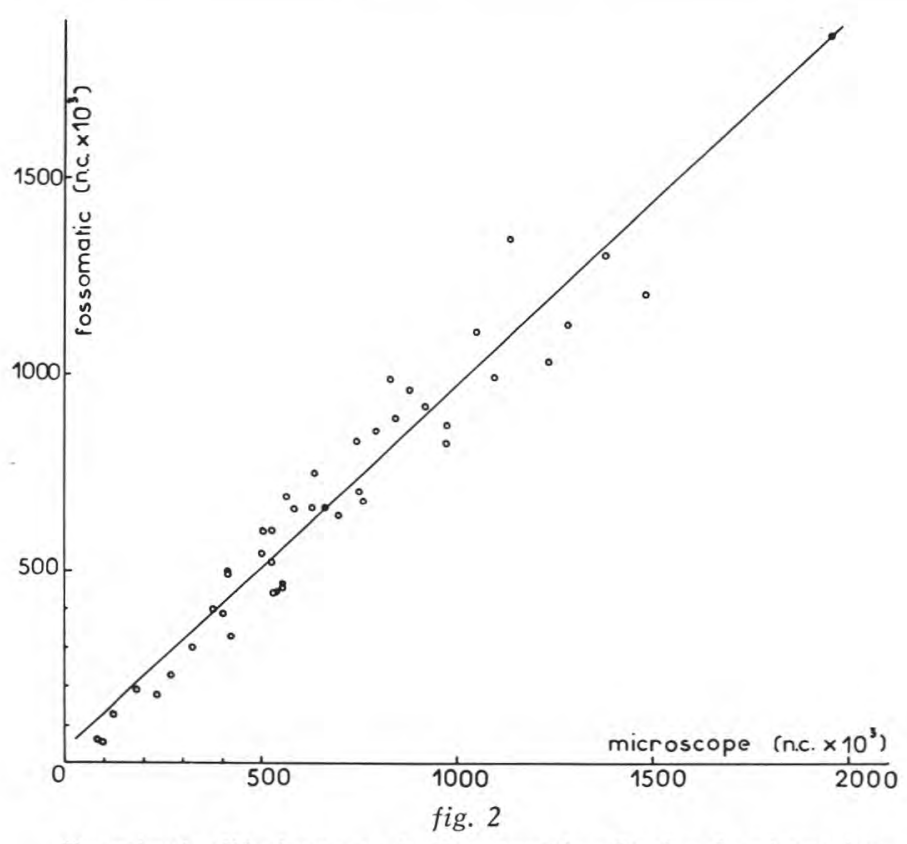

Exactitude d'étalonnage du Fossomatic : Droite de régression des comptages des nombres de cellules (n.c.) effectués avec le Fossomatic au seuil de 1,5 en fonction des comptages microscopiques.

$\mathrm{n}=44 ; \mathrm{y}=0,94 \mathrm{x}+34 ; \mathrm{r}=0,976 ; \mathrm{x}=728 ; \mathrm{y}=720$.

1.21. Influence de l'addition d'un produit de conservation au lait sur la courbe de seuil

Les courbes de seuil du MCC montrent que l'addition de $\mathrm{Cr}_{2} \mathrm{O}_{7} \mathrm{~K}_{2}$ ou de $\mathrm{NaN}_{3}$ n'exerce pas d'influence importante sur l'allure de la courbe et la position du plateau. En revanche, avec $\mathrm{HgCl}_{2}$, on observe une courbe plus aplatie et les résultats sont supérieurs de 20 p. 100 environ, au niveau du plateau, aux résultats obtenus sur le lait sans conservateur. Ce conservateur a été de ce fait éliminé pour le compteur Coulter.

Les courbes de seuil correspondant à la méthode de Tolle n'ont pas été réalisées.

\subsection{Comparaison avec les comptages microscopiques}

L'analyse porte sur 26 échantillons de lait de troupeau renfermant entre 250000 et 2,0 millions de cellules/ml la limite inférieure est imposée par le fait que le Compteur Coulter donne des résultats surestimés pour les échantillons ayant moins de 250000 cellules $/ \mathrm{ml}$ ).

- Les mesures avec le MCC ont été faites à différents seuils compris entre 7 et 11 correspondant au " plateau " de la courbe de seuil. Les résultats du tableau 1 montrent que la précision par 
TABLEAU 1

Précision par rapport à la méthode microscopique et exactitude d'étalonnage du MCC en fonction du seuil ( $\mathrm{n}=26$ échantillons)

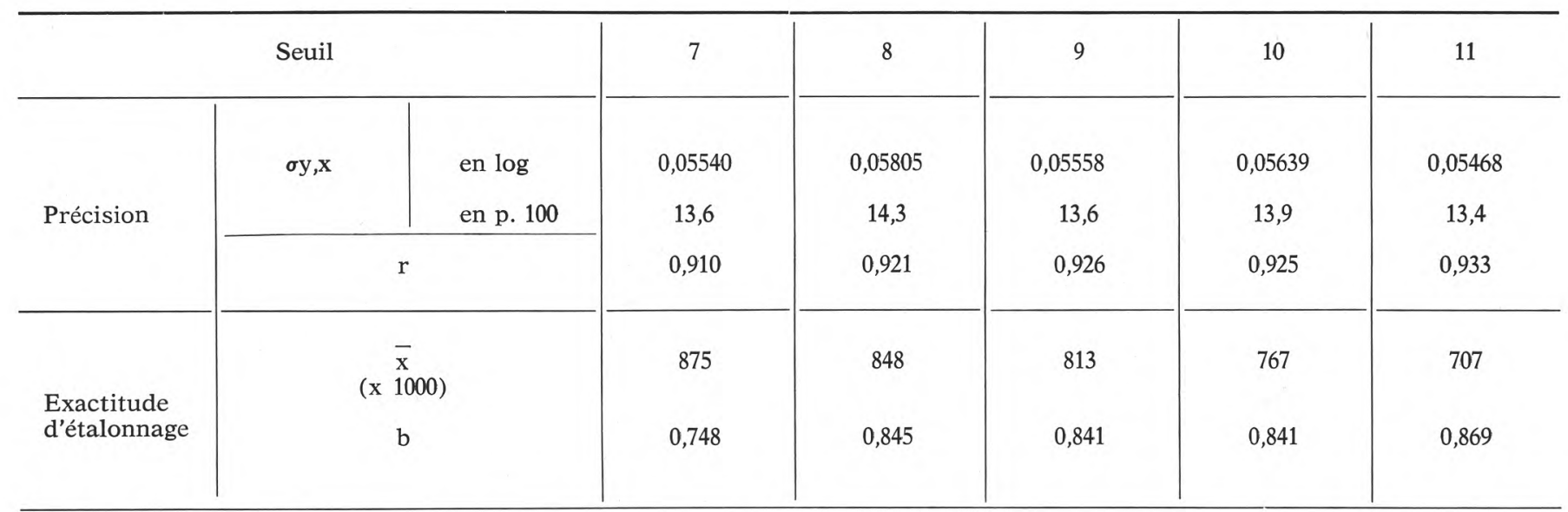

$\sigma \mathrm{y}, \mathrm{x}$ : écart-type résiduel déduit de la régression $\mathrm{MCC} /$ microscope. $\mathrm{b}$ : coefficient de régression $\mathrm{MCC} / \mathrm{microscope}$.

$r$ : coefficient de corrélation.

Moyenne géométrique des comptages microscopiques : 794000

$\mathrm{x}$ : moyenne géométrique des comptages MCC. cellules/ml. 


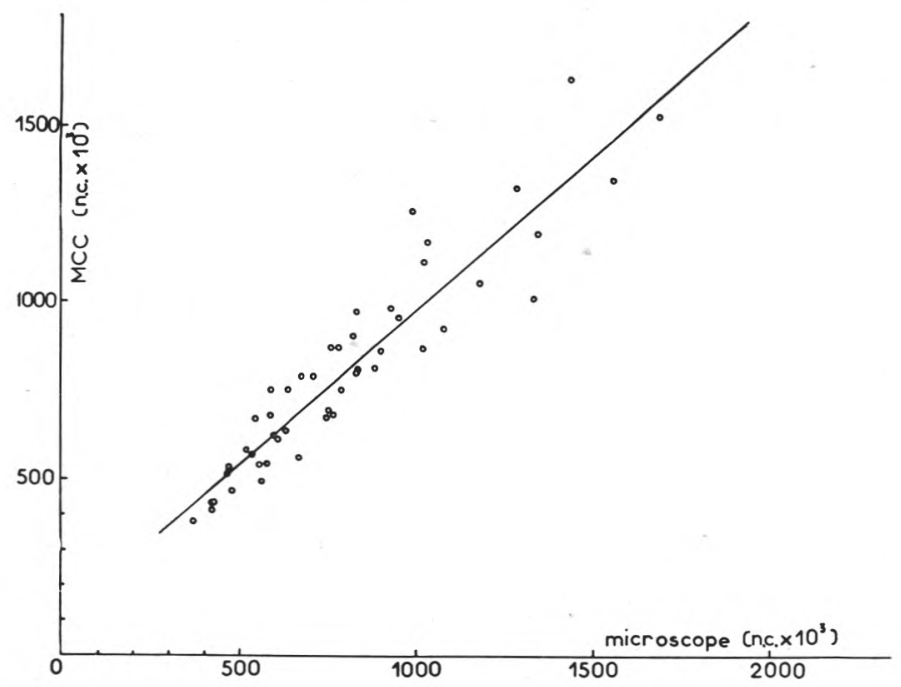

fig. 3

Exactitude d'étalonnage du MCC : Droite de régression des comptages des nombres de cellules (n.c.) effectués avec le MCC au seuil de $9(4,5 \mu \mathrm{m})$ en fonction des comptages microscopiques.

$\mathrm{n}=49 ; \mathrm{y}=0,87 \mathrm{x}+107 ; \mathrm{r}=0,933 ; \overline{\mathrm{x}}=799 ; \overline{\mathrm{y}}=801$.

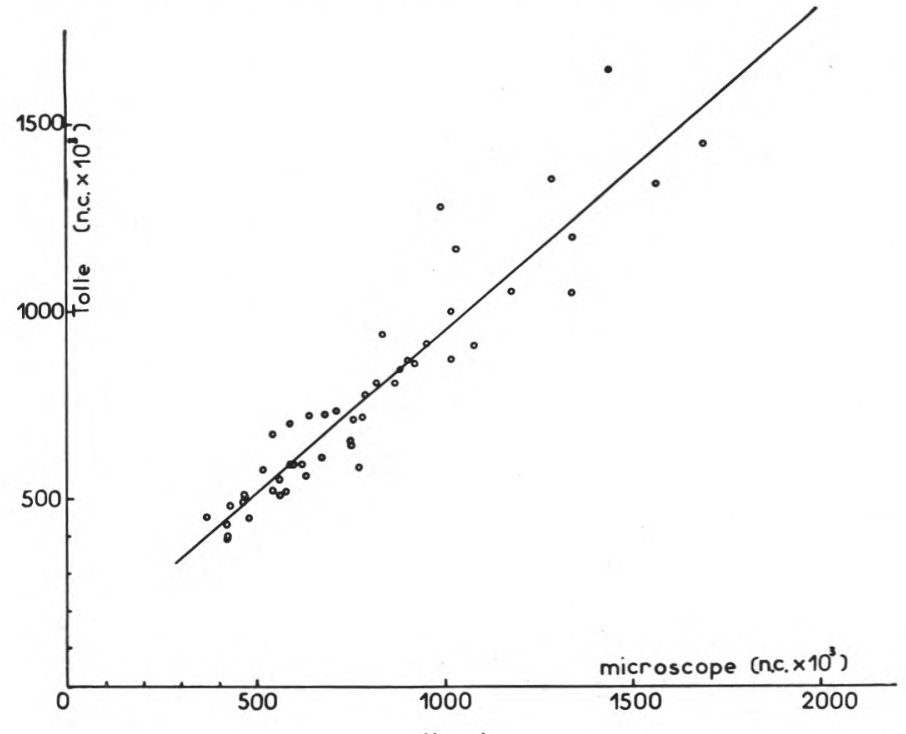

fig. 4

Exactitude d'étalonnage de la méthode de Tolle : Droite de régression des comptages des nombres de cellules (n.c.) effectués par la méthode de Tolle au seuil de $4,6 \mu \mathrm{m}$ en fonction des comptages microscopiques.

$\mathrm{n}=49 ; \mathrm{y}=0,87 \mathrm{x}+81 ; \mathrm{r}=0,939 ; \overline{\mathrm{x}}=799 ; \overline{\mathrm{y}}=779$. 
rapport aux comptages microscopiques est, dans les limites fixées, indépendante du seuil. C'est entre les seuils de 9 et 10 correspondant à un diamètre calculé légèrement supérieur à $4,5 \mu \mathrm{m}$ que devrait normalement se situer le seuil de lecture.

- Les analyses par la méthode de Tolle effectuées au seuil correspondant à un diamètre de $4,6 \mu \mathrm{m}$ ont donné une moyenne identique à celle des comptages microscopiques et une précision semblable à celle du MCC $\left(\sigma_{y, \mathrm{x}}=13,1 \mathrm{p} .100\right)$.

Les figures 3 (MCC) et 4 (méthode de Tolle) portant sur 53 échantillons examinés respectivement aux seuils de 4,5 et 4,6 $\mu \mathrm{m}$ définis plus haut, confirment ces résultats.

Dans les deux cas (MCC, méthode de Tolle), l'addition de $\mathrm{Cr}_{2} \mathrm{O}_{7} \mathrm{~K}_{2}$ ou de $\mathrm{NaN}_{3}$ ne modifie pas la précision des comptages par rapport à la méthode microscopique.

\section{2) Conservation des échantillons}

Nous avons déjà signalé que, dans la majorité des cas, les échantillons utilisés pour effectuer des numérations cellulaires sont prélevés en vue d'une autre utilisation : détermination de la composition chimique pour le contrôle laitier individuel, détermination de la qualité bactériologique ou de la composition chimique des laits livrés par les producteurs en vue du paiement du lait selon sa qualité. Il s'ensuit que les conditions de conservation de ces échantillons sont souvent très différentes, l'analyse pouvant être faite aussi bien sur des échantillons de lait frais sans conservateur que sur des échantillons additionnés de conservateur et âgés de plusieurs jours voire de plusieurs semaines et gardés tantôt à la température extérieure et tantôt au froid. Une attention toute particulière a donc été portée à ces problèmes notamment à la conservation des échantillons avec $\mathrm{Cr}_{2} \mathrm{O}_{7} \mathrm{~K}_{2}$ qui est le produit le plus largement employé.

\subsection{Fossomatic}

\subsection{Nature du produit de conservation}

L'étude des courbes de seuil effectuée au paragraphe précédent a montré qu'au seuil de 1,5, il n'existe aucune différence importante entre les résultats obtenus sur les échantillons de lait additionnés ou non de l'un quelconque des trois produits de conservation envisagés.

\subsection{Durée et température de conservation}

Pour les différents conservateurs, nous avons étudié l'influence de la durée et de la température $\left(4^{\circ} \mathrm{C}\right.$ ou $\left.20^{\circ} \mathrm{C}\right)$ de conservation des échantillons de lait ainsi que l'influence du cumul mensuel des échantillons.

L'analyse statistique des résultats a été faite séparément pour chaque conservateur. Elle porte sur la comparaison, par la méthode 
des couples, des moyennes géométriques des résultats des analyses faites sur les mêmes échantillons, d'une part le jour de la traite (référence) et d'autre part, après conservation dans des conditions définies; la différence entre ces deux moyennes géométriques est exprimée en valeur relative (tab. 2 ).

\subsection{Conservation à $4^{\circ} \mathrm{C}$}

- Pour les échantillons de lait sans conservateur, les analyses peuvent être faites jusqu'à 3 à $4 \mathrm{j}$ de conservation sans modification significative des résultats.

- Les échantillons additionnés de $\mathrm{HgCl}_{2}$ peuvent être analysés au moins jusqu'à $7 \mathrm{j}$ de conservation.

Dans le cas de $\mathrm{Cr}_{2} \mathrm{O}_{7} \mathrm{~K}_{2}$, l'étude ayant été poursuivie jusqu'à $23 \mathrm{j}$, pendant cette période, les résultats montrent qu'il n'y a aucune influence significative de la durée de conservation à $4^{\circ} \mathrm{C}$ sur les dosages avec le Fossomatic.

- $\mathrm{NaN}_{3}$ semble être un conservateur beaucoup moins efficace puisqu'on observe une baisse significative des résultats de 8,9 p. 100 en moyenne après $1 \mathrm{j}$ de conservation. D'une manière générale, la baisse des résultats s'accompagne d'une augmentation de la variance des écarts entre les résultats de la série de référence et les résultats de la série analysée après conservation. Cette augmentation de la variance indique une influence variable de la conservation suivant les échantillons de lait. Les différences, trouvées non significatives au test $t$ sur les échantillons âgés de 3-4 j et 5-7 j, peuvent certainement être expliquées par cette augmentation de la variance.

\subsection{Conservation à $20^{\circ} \mathrm{C}$}

Sauf dans le cas des échantillons conservés avec $\mathrm{HgCl}_{2}$, la conservation à $20^{\circ} \mathrm{C}$ entraîne une sous-estimation significative des résultats. Dans le cas des échantillons conservés avec $\mathrm{Cr}_{2} \mathrm{O}_{7} \mathrm{~K}_{2}$, cette sous-estimation faible après $2 \mathrm{j}$ de conservation, atteint 9 p. 100 après 1 semaine. La baisse des résultats est très nette lorsque les échantillons sont conservés avec NaN.

\subsection{Cumul mensuel des échantillons}

Le cumul des échantillons, pratiqué dans certaines régions, consiste à prélever trois fois par mois un échantillon de lait et à effectuer l'analyse en fin de mois sur l'échantillon de lait cumulé comprenant le mélange des trois échantillons partiels. L'échantillon cumulé est gardé à $4^{\circ} \mathrm{C}$ en présence de $\mathrm{K}_{2} \mathrm{Cr}_{2} \mathrm{O}_{7}$.

Afin de tester l'influence du cumul d'échantillons, nous avons examiné le jour de leur prélèvement, les trois échantillons partiels constituant le cumul, pour comparer ensuite la moyenne arithmétique de ces trois résultats (constituant la valeur de référence) avec le résultat obtenu sur l'échantillon cumulé après conservation. Deux séries de douze et seize échantillons ont ainsi été examinées. La première série a porté sur des échantillons cumulés provenant de 
TABLEAU 2

Influence de la durée et de la température de conservation des échantillons de lait sur les analyses faites avec le Fossomatic

A : Conservation à $4^{\circ} \mathrm{C}$

\begin{tabular}{|c|c|c|c|c|c|c|c|c|}
\hline \multirow{2}{*}{$\begin{array}{c}\text { Durée de } \\
\text { conservation } \\
\text { (jour) }\end{array}$} & \multicolumn{2}{|c|}{$\begin{array}{c}\text { Sans } \\
\text { conservateur }\end{array}$} & \multicolumn{2}{|c|}{$\mathrm{K}_{2} \mathrm{Cr}_{2} \mathrm{O}_{7}$} & \multicolumn{2}{|c|}{$\mathrm{HgCl}_{2}$} & \multicolumn{2}{|c|}{$\mathrm{NaN}_{3}$} \\
\hline & $\mathrm{n}$ & d & $\mathrm{n}$ & d & $\mathrm{n}$ & d & $\mathrm{n}$ & d \\
\hline 1 & 27 & $+0,3$ p. 100 & 30 & $-1,1$ p. 100 & 15 & $-2,8$ p. 100 & 15 & $-8,9$ p. $100^{* *}$ \\
\hline 2 & 15 & $+2,5$ p. 100 & - & - & - & - & - & - \\
\hline $3-4$ & 12 & $-2,5$ p. 100 & 55 & $-0,4$ p. 100 & 27 & $-1,8$ p. 100 & 27 & $-3,6$ p. 100 \\
\hline $5-7$ & - & - & 12 & $-1,5$ p. 100 & 12 & $-1,2$ p. 100 & 12 & $-6,2$ p. 100 \\
\hline 8-14 & - & - & 34 & $-1,2$ p. 100 & - & - & - & - \\
\hline $15-21$ & - & - & 28 & $-2,4$ p. 100 & - & - & - & - \\
\hline 23 & - & - & 12 & $-1,3$ p. 100 & - & - & - & - \\
\hline
\end{tabular}


$\mathrm{B}$ : conservation à $20^{\circ} \mathrm{C}$

\begin{tabular}{|c|c|c|c|c|c|c|}
\hline \multirow{2}{*}{$\begin{array}{l}\text { Durée de } \\
\text { conservation } \\
\text { (jour) }\end{array}$} & \multicolumn{2}{|c|}{$\mathrm{K}_{2} \mathrm{Cr}_{2} \mathrm{O}_{7}$} & \multicolumn{2}{|c|}{$\mathrm{HgCl}_{2}$} & \multicolumn{2}{|c|}{$\mathrm{NaN}_{3}$} \\
\hline & $\mathrm{n}$ & d & $\mathrm{n}$ & d & $\mathrm{n}$ & d \\
\hline 1 & 27 & $-1,1$ p. 100 & 27 & $-1,7$ p. 100 & 27 & $-6,0$ p. $100^{* *}$ \\
\hline 2 & 15 & $-3,1$ p. $100^{* * *}$ & 15 & $-0,7$ p. 100 & 15 & $-13,3$ p. $100^{* *}$ \\
\hline $3-4$ & 27 & $-11,8$ p. $100^{* *}$ & 27 & $-5,3$ p. $100^{*}$ & 27 & $-14,8$ p. $100^{* *}$ \\
\hline $5-7$ & 58 & $-6,2$ p. $100^{*}$ & 12 & $-3,9$ p. 100 & 12 & $-28,4$ p. $100^{*}$ \\
\hline
\end{tabular}

$\mathrm{n}=$ nombre d'échantillons analysés.

$\mathrm{d}=$ différence moyenne des comptages (exprimée en valeur relative des nombres) par rapport aux analyses effectuées le jour de la traite.

* : différence significative $(P<0,05)$.

** : différence hautement significative $(P<0,01)$. 


\section{TABLEAU 3}

Influence de la durée et de la température de conservation des échantillons de lait sur les analyses faites avec le Compteur Coulter, par la méthode automatique (MCC) et par la méthode de Tolle ( $\mathrm{n}=32$ échantillons)

\begin{tabular}{|c|c|c|c|c|c|c|}
\hline \multirow{2}{*}{ Méthode } & \multirow{2}{*}{$\begin{array}{c}\text { Durée de } \\
\text { conservation } \\
\text { (jour) }\end{array}$} & \multicolumn{3}{|c|}{ Conservation à $4^{\circ} \mathrm{C}$} & \multicolumn{2}{|c|}{ Conservation à $20^{\circ} \mathrm{C}$} \\
\hline & & $\begin{array}{c}\text { Sans } \\
\text { conservateur }\end{array}$ & $\mathrm{K}_{2} \mathrm{Cr}_{2} \mathrm{O}_{7}$ & $\mathrm{NaN}_{3}$ & $\mathrm{~K}_{2} \mathrm{Cr}_{2} \mathrm{O}_{7}$ & $\mathrm{NaN}_{3}$ \\
\hline \multirow{5}{*}{ MCC } & 1 & $-1,0$ & $+1,4$ & $-1,6$ & $-0,9$ & $-4,5^{\star *}$ \\
\hline & 2 & $+0,9$ & $+1,8$ & $+1,4$ & $-5,8^{* *}$ & $-6,5^{* *}$ \\
\hline & 3 & $+4,6^{* *}$ & $+2,8^{*}$ & $-0,9$ & $-5,5^{* *}$ & $-7,9 * *$ \\
\hline & 6 & - & $+0,3$ & $-2,3$ & $-0,2$ & $-5,9^{*}$ \\
\hline & 14 & - & $+0,6$ & $-3,8$ & - & - \\
\hline \multirow{5}{*}{$\begin{array}{c}\text { Méthode } \\
\text { de } \\
\text { Tolle }\end{array}$} & 1 & $+1,1$ & - & - & $-1,2$ & - \\
\hline & 2 & $+0,4$ & $+0,7$ & - & $-6,4^{* *}$ & - \\
\hline & 3 & $+4,4$ & $-0,9$ & - & $-5,5^{* *}$ & - \\
\hline & 6 & - & $-11,4^{* *}$ & - & $-11,4^{\star *}$ & - \\
\hline & 14 & - & $-12,6$ & - & - & - \\
\hline
\end{tabular}

Différences moyennes, exprimées en valeur relative (en p. 100) des nombres, par rapport aux analyses faites le jour de la traite.

* différence significative $(\mathrm{P}<0,05)$.

** différence hautement significative $(P<0,01)$.

(a) pour la méthode Tolle, référence au jour $\mathrm{J}+1$ à $4^{\circ} \mathrm{C}$. 
prélèvements effectués 4,17 et 23 j avant l'examen et la deuxième série sur des prélèvements effectués 3,11 et $17 \mathrm{j}$ avant l'examen. Le cumul d'échantillons s'est traduit, au niveau de la moyenne géométrique, par une baisse de 6,8 p.100 ( $\mathrm{P}<0,01)$ uniquement pour la première série ; pour la deuxième série, la différence n'est pas significative.

\subsection{Compteur Coulter}

L'étude a été limitée à deux conservateurs $\left(\mathrm{K}_{2} \mathrm{Cr}_{2} \mathrm{O}_{7}\right.$ et $\left.\mathrm{NaN}_{3}\right)$ et la durée de conservation des échantillons n'a pas dépassé $14 \mathrm{j}$. Le problème du cumul des échantillons n'a pas été étudié.

Les essais ont porté sur deux séries de quinze et dix-sept échantillons de lait ayant entre 200000 et 2 millions de cellules/ml. L'analyse statistique est identique à celle qui a été employée dans le cas du Fossomatic (§ 3.12).

\subsection{Nature du produit de conservation}

MCC : l'addition de $\mathrm{K}_{2} \mathrm{Cr}_{2} \mathrm{O}_{7}$ entraîne une diminution non significative des résultats de 1,0 p. 100 , celle de $\mathrm{NaN}_{3}$, une diminution faible mais significative de 2,7 p. 100.

Méthode de Tolle : l'addition de $\mathrm{K}_{2} \mathrm{Cr}_{2} \mathrm{O}_{7}$ entraîne une augmentation significative de 2,6 p. $100 ; \mathrm{NaN}_{3}$ diminuant les résultats de 12,7 p. $100(\mathrm{P}<0,01)$ a été éliminé pour la méthode de Tolle.

\subsection{Durée et température de conservation}

Les limites de conservation à $4^{\circ} \mathrm{C}$ et $20^{\circ} \mathrm{C}$ ont été respectivement de 14 et 6 j (tab. 3).

\subsection{Conservation à $4^{\circ} \mathrm{C}$}

- $M C C$ : Les échantillons gardés sans conservateur peuvent être examinés jusqu'à $48 \mathrm{~h}$ après la traite. Ensuite, on observe une augmentation significative de 4,6 p.100 au $3^{\text {e }}$ jour de conservation.

L'addition de $\mathrm{Cr}_{2} \mathrm{O}_{7} \mathrm{~K}_{2}$ ou de $\mathrm{NaN}_{3}$ permet de conserver les échantillons jusqu'à $14 \mathrm{j}$ sans modification importante des résultats.

- Méthode de Tolle: D’une manière générale, les possibilités de conservation sont plus limitées qu'avec le MCC. Si les échantillons non additionnés de conservateur peuvent être analysés jusqu'à $48 \mathrm{~h}$ après la traite, la conservation des échantillons additionnés de $\mathrm{K}_{3} \mathrm{Cr}_{2} \mathrm{O}_{7}$ doit être limitée à $3 \mathrm{j}$ environ. Au-delà, la baisse des résultats est très importante.

\subsection{Conservation à $20^{\circ} \mathrm{C}$}

- $M C C$ : Avec $\mathrm{NaN}_{3}$ et $\mathrm{K}_{2} \mathrm{Cr}_{2} \mathrm{O}_{7}$, on constate dès le $1^{\text {er }}$ jour de conservation, une baisse des résultats qui oscille entre 4 et 8 p. 100 .

- Méthode Tolle : La conservation des échantillons additionnés de $\mathrm{Cr}_{2} \mathrm{O}_{7} \mathrm{~K}_{2}$ s'accompagne d'une baisse régulière qui atteint $-11,4$ p. 100 après $6 \mathrm{j}$ de conservation. 


\section{DISCUSSION}

1) Compte tenu du fait que la plupart des laboratoires utilisateurs n'ont pas la possibilité de contrôler eux-mêmes l'exactitude des résultats fournis par leur appareil, les moyens à mettre en œuvre pour effectuer ce contrôle revêtent une grande importance, et ceci, d'autant plus que des erreurs importantes peuvent être commises sans que l'utilisateur s'en aperçoive puisqu'il ne dispose pas actuellement de références précises concernant le nombre de cellules, de laits individuels ou de laits de troupeau qu'il doit examiner.

Pour garantir la précision des numérations cellulaires, il y a deux aspects importants à considérer : l'étalonnage initial des appareils et le contrôle régulier des résultats fournis par l'appareil.

Quelle que soit la méthode employée, l'étalonnage initial doit être réalisé par le constructeur, sur la base des résultats fournis par plusieurs laboratoires spécialisés ayant déterminé le seuil de lecture avec la méthode microscopique par comparaison.

Dans le cas du Compteur Coulter, le seuil de lecture est relié à une grandeur mesurable : le diamètre apparent (ou le volume) des particules. Si le diamètre est bien défini, d'après nos propres résultats il est situé entre 4,5 et 4,6 $\mu \mathrm{m}$, aussi bien avec la méthode de Tolle (Grappin et Jeunet, 1971) qu'avec le MCC, il est alors très facile de procéder à un étalonnage des appareils à l'aide de particules étalons.

Pour le Fossomatic, la situation est moins simple puisque le seuil correspond cette fois à une intensité fluorescente plus difficile à mesurer. Si la valeur de seuil de 1,5 que nous avons définie ne peut pas être étendue aux autres appareils car c'est une caractéristique propre à chaque appareil, en revanche, l'intensité de la lumière fluorescente (ou signal) correspondant à ce seuil pourrait être utilisée par le constructeur pour définir le seuil de chaque appareil.

Le réglage de seuil d'un appareil peut être correct sans que, pour autant, les résultats soient exacts. En effet, les réactifs, le mode opératoire, le réglage des seringues, etc. peuvent être défecteux et entraîner des résultats inexacts. Il faut donc que l'utilisateur puisse vérifier, outre l'étalonnage de son appareil (seuil) l'ensemble des réactifs et des opérations, qu'elles soient effectuées manuellement (cas du Coulter avec la méthode de Tolle) ou automatiquement (MCC, Fossomatic). Pour cela, il faudrait que les laboratoires disposent d'un ou plusieurs étalons permettant de vérifier fréquemment la justesse de leurs résultats.

Deux solutions peuvent être envisagées pour la fourniture d'étalons :

- Utilisation de suspension de leucocytes lyophilisées de concentration connue, accompagnées d'un lait lyophilisé contenant peu 
de cellules et destiné à vérifier l'efficacité des réactifs. De tels étalons existent en Allemagne (laboratoire de l'Institut de Recherches Laitières de Kiel) et donnent de très bons résultats avec la méthode de Tolle et probablement, sous réserve de confirmation, avec le MCC. Par contre, ces suspensions ne sont pas utilisables actuellement avec le Fossomatic.

- Distribution par un laboratoire central de plusieurs échantillons de lait analysés par la méthode microscopique. Ces échantillons pourraient éventuellement être additionnés de $\mathrm{Cr}_{3} \mathrm{O}_{7} \mathrm{~K}_{2}$ et acheminés rapidement de façon à ce que l'analyse puisse être effectuée au plus tard $48 \mathrm{~h}$ après le prélèvement. L'emploi d'emballages isothermes permettant de garder les échantillons entre $4^{\circ} \mathrm{C}$ et $10^{\circ} \mathrm{C}$ est indispensable.

Mais à côté de ces vérifications concernant la justesse des résultats, il est indispensable, comme pour toute détermination en série, de contrôler la fidélité de l'appareil. Dans ce but, l'utilisation d'un lait de contrôle inclus régulièrement dans la chaîne d'analyse (tous les 20-30 échantillons) est une très bonne formule. En vue de ce contrôle, nous avons montré qu'il est possible de garder des échantillons de lait additionnés de dichromate de potassium pendant 7 à $14 \mathrm{j}$ (Fossomatic, MCC) ou $3 \mathrm{j}$ (méthode de Tolle) et de constituer ainsi une réserve d'échantillons de lait de contrôle qui peuvent être utilisés pendant la période considérée. La teneur en cellules de ce lait doit être déterminée par comparaison avec l'étalon ou, le cas échéant, avec le lait de contrôle précédent.

2) Nous avons déjà souligné qu'il n'était souvent pas possible aux laboratoires de faire des numérations leucocytaires sur du lait frais moins de 24 ou $48 \mathrm{~h}$ après la traite. Aussi, l'utilisateur doit-il connaître exactement les limites et les conditions de conservation des échantillons compatibles avec l'emploi des différentes techniques.

Nous avons résumé dans le tableau 4 les différentes possibilités de conservation des échantillons de lait en ne retenant comme conservateur que les produits pour lesquels on a observé une influence systématique inférieure à 5 p. 100 .

On peut retenir de ce tableau que parmi les trois méthodes d'analyse étudiées, c'est le Fossomatic qui offre la plus grande souplesse d'utilisation et que le MCC permet l'examen d'échantillons plus âgés que la méthode de Tolle.

Parmi les produits de conservation, le dichromate de potassium est le seul conservateur pouvant être utilisé sans trop d'inconvénients avec les trois méthodes d'analyses.

Lorsque les dénombrements leucocytaires sont faits sur des échantillons de lait prélevés dans le cadre du contrôle laitier individuel, les échantillons sont obligatoirement additionnés d'un conservateur $\left(\mathrm{K}_{2} \mathrm{Cr}_{2} \mathrm{O}_{i}, \mathrm{HgCl}_{2}\right.$ et plus rarement $\left.\mathrm{NaN}_{3}\right)$ et sont parfois gardés à la température extérieure pendant 3 à $8 \mathrm{j}$ avant d'être analysés. 
TABLEAU 4

Conditions de conservation des échantillons de lait utilisés pour les numérations cellulaires

\begin{tabular}{|c|c|c|c|c|c|c|c|}
\hline \multicolumn{3}{|c|}{$\begin{array}{c}\text { Nature } \\
\text { du conservateur }\end{array}$} & $\begin{array}{c}\text { Sans } \\
\text { conservateur }\end{array}$ & $\mathrm{HgCl}_{2}$ & $\mathrm{~K}_{2} \mathrm{Cr}_{2} \mathrm{O}_{7}$ & $\mathrm{NaN}_{3}$ & HCHO (a) \\
\hline \multicolumn{3}{|c|}{ Dose } & - & 0,07 p. 100 & 0,1 p. 100 & 0,03 p. 100 & 0,07 p. 100 \\
\hline$P$ & & $\begin{array}{l}\text { ssomatic } \\
\text { MCC } \\
\text { Tolle }\end{array}$ & $\begin{array}{l}\text { oui } \\
\text { oui } \\
\text { oui }\end{array}$ & $\begin{array}{l}\text { oui } \\
\text { non } \\
\text { non }\end{array}$ & $\begin{array}{l}\text { oui } \\
\text { oui } \\
\text { oui }\end{array}$ & $\begin{array}{l}\text { non } \\
\text { oui } \\
\text { non }\end{array}$ & $\begin{array}{l}\text { non } \\
\text { oui } \\
\text { oui }\end{array}$ \\
\hline $\begin{array}{l}\text { Durée } \\
\text { limite } \\
\text { de } \\
\text { conservation } \\
\text { (en jour) }\end{array}$ & $4^{\circ} \mathrm{C}$ & $\begin{array}{c}\text { Fossomatic } \\
\text { MCC } \\
\text { Tolle } \\
\text { Fossomatic } \\
\text { MCC } \\
\text { Tolle }\end{array}$ & $\begin{array}{l}3 \\
2 \\
2\end{array}$ & $\begin{array}{l}2 \\
- \\
-\end{array}$ & $\begin{array}{c}14-21 \\
7-14 \\
3 \\
2 \\
1 \\
1\end{array}$ & $\begin{array}{l}- \\
7-14 \\
-\end{array}$ & $\begin{array}{l}- \\
\text { nd } \\
14 \\
- \\
\text { nd } \\
7\end{array}$ \\
\hline
\end{tabular}

nd: non déterminé.

(a) : le formol est considéré ici comme produit de conservation des échantillons et non comme agent de fixation des cellules. (b) : d'après " A Monograph on bovine mastitis - Part I (1971), Annual Bulletin F.I.L. ». 
Dans de telles conditions, aucune méthode ne permet d'obtenir avec certitude des résultats exacts. Même en excluant l'azide de sodium dans le cas du Fossomatic, on constate, avec les autres conservateurs et pour toutes les méthodes, que les résultats sont en moyenne sous-estimés de 10 p. 100 environ avec d'assez larges variations individuelles. De telles erreurs sont-elles compatibles avec une prophylaxie individuelle des mammites ? Sachant que, dans la majorité des départements, il est impossible de faire au niveau individuel un prélèvement spécial pour les comptages leucocytaires, peut-on se passer de cette source d'information sous prétexte que les résultats sont entâchés d'une certaine erreur ?

La même question se pose dans le cas de contrôles faits sur certains échantillons destinés aux analyses en vue du paiement du lait à la qualité, notamment dans le cas des échantillons cumulés.

En raison de la variabilité individuelle ou par troupeau du nombre de cellules du lait, l'erreur provenant de la conservation des échantillons reste relativement faible et dans des limites acceptables; les renseignements fournis par la numération demeurent certainement valables dans un but de prophylaxie.

Toutefois, s'il s'agit d'effectuer un paiement en fonction du nombre de cellules, ou de contrôler la qualité hygiénique, il faut limiter impérativement les analyses aux échantillons gardés dans des conditions ne modifiant pas de façon significative les résultats d'analyse. De toute façon, il nous paraît extrêmement important de souligner que l'analyse d'échantillons âgés de $3 \mathrm{j}$ ou plus n'est qu'un pis-aller dont il faut bien connaître les inconvénients : erreurs systématiques plus ou moins importantes selon le conservateur employé et dont il faudrait tenir compte au moment de l'étalonnage, et risque d'erreur accru d'une part, lorsque la qualité bactériologique des échantillons n'est pas satisfaisante et d'autre part lorsque ces échantillons sont gardés longtemps à une température élevée.

\section{R és u mé}

Cette étude porte :

$1^{\text {o }}$ Sur l'influence des conditions de conservation des échantillons de lait (nature du produit de conservation : $\mathrm{K}_{2} \mathrm{Cr}_{2} \mathrm{O}_{7}, \mathrm{HgCl}_{2}, \mathrm{NaN}_{3}$, durée et température de conservation) sur la précision des comptages cellulaires effectués par les appareils Compteur Coulter (avec le réactif de Tolle), Milk Cell Counter (avec le réactif Somesol) et Fossomatic.

$2^{\circ}$ Sur les modalités pratiques d'étalonnage et de contrôle de ces appareils. 
Parmi les conservateurs étudiés seul $\mathrm{Cr}_{2} \mathrm{O}_{7} \mathrm{~K}_{2}$ peut être employé avec les trois méthodes. Dans ce cas la durée de conservation à $20^{\circ} \mathrm{C}$ doit être limitée à $2 \mathrm{j}$; à $4^{\circ} \mathrm{C}$ la durée varie avec la méthode employée, elle peut atteindre 3 semaines dans le cas du Fossomatic.

L'étalonnage basé uniquement sur le diamètre correspondant au seuil de lecture (Compteur Coulter) ou sur la forme de la courbe de seuil (Fossomatic) est insuffisant; dans tous les cas il est recommandé de se référer régulièrement à la méthode microscopique directe.

\section{S u $\mathbf{m} \mathbf{m}$ a r y}

This study deals with :

$1^{\text {o }}$ The influence of keeping conditions of milk samples (kind of preservatives : $\mathrm{HgCl}_{2}, \mathrm{~K}_{2} \mathrm{Cr}_{2} \mathrm{O}_{4}, \mathrm{NaN}_{3}$; température and duration of storage) on the accuracy of the somatic cell's counts obtained with the Coulter Counter (with Tolle's reagent), Milk Cell Counter (with Somasol reagent) and Fossomatic.

$2^{\circ}$ The practical conditions of calibration and control of these instruments.

Between the différent preservatives only $\mathrm{K}_{2} \mathrm{Cr}_{2} \mathrm{O}_{7}$ can be used with the 3 instruments. In this case the time of storage must be limited to 2 days at $20^{\circ} \mathrm{C}$; at $4^{\circ} \mathrm{C}$ it varies with the method employed, cells determination can be made up to 3 weeks of storage with the Fossomatic.

Calibration founded only on the threshold value corresponding to a fixed particle diameter (Coulter) or on the shape of the "threshold curve " (Fossomatic) is insufficent, for all the instruments a frequent reference to the direct microscopic method is recommended.

Reçu pour publication en juin 1975.

\section{Références bibliographiques}

Grappin (R.) et Jeunet (R.) (1971). - Essais de l'appareil "Compteur Coulter " utilisé pour la détermination du nombre de cellules totales des laits de troupeaux. Le Lait, 51, 273-293.

Grappin (R.) et Jeunet (R.) (1973). - Essai d'une chaîne automatique de numération des cellules du lait utilisant le Compteur Coulter. Rev. Lait. Franç., $\mathrm{n}^{\circ} 313,737-747$.

Grappin (R.) et JeUnet (R.) (1974). - Premiers essais de l'appareil "Fossomatic " pour la détermination automatique du nombre de cellules du lait. Le Lait, $54,627-644$.

SchmidT-MadSEN (P.) (1975). - Fluoro-opto-electronic cell-counting on milk. J. Dairy Res., 42, 227-239.

Zeidler (H.), Tolle (A.) und Heeschen (W.) (1968). - Verbesserte Präparationstechnik zur elektronischen Bestimmung des Zellgehaltes in Milch. Milchwissenschaft, 23, 564-568. 